\title{
Functional remediation in bipolar disorder: 1-year follow-up of neurocognitive and functional outcome
}

\author{
C. M. Bonnin, C. Torrent, C. Arango, B. L. Amann, B. Solé, A. González-Pinto, J. M. Crespo, \\ R. Tabarés-Seisdedos, M. Reinares, J. L. Ayuso-Mateos, M. P. García-Portilla, Á. Ibañez, \\ M. Salamero, E. Vieta, A. Martinez-Aran and the CIBERSAM Functional Remediation Group
}

\section{Background}

Few randomised clinical trials have examined the efficacy of an intervention aimed at improving psychosocial functioning in bipolar disorder.

\section{Aims \\ To examine changes in psychosocial functioning in a group that has been enrolled in a functional remediation programme 1 year after baseline.}

\section{Method}

This was a multicentre, randomised, rater-masked clinical trial comparing three patient groups: functional remediation, psychoeducation and treatment as usual over 1-year follow-up. The primary outcome was change in psychosocial functioning measured by means of the Functioning Assessment Short Test (FAST). Group $\times$ time effects for overall psychosocial functioning were examined using repeated-measures ANOVA (trial registration NCT01370668).

\section{Results}

There was a significant group $\times$ time interaction for overall psychosocial functioning, favouring patients in the functional remediation group $(F=3.071$, d.f. $=2, P=0.049$ ).

\section{Conclusions}

Improvement in psychosocial functioning is maintained after 1-year follow-up in patients with bipolar disorder receiving functional remediation.

\section{Declaration of interest}

None.

\section{Copyright and usage}

(c) The Royal college of Psychiatrists 2016.
Functional impairment in patients with bipolar disorder is common even during periods of euthymia. ${ }^{1}$ Longitudinal and cross-sectional reports in patients with bipolar disorder confirm that neurocognitive and psychosocial functioning are related to each other ${ }^{2-4}$ and that neurocognitive performance can predict functional outcome. ${ }^{3,5,6}$ Given the link between neurocognition and functional outcome $\mathrm{e}^{3-6}$ the functional remediation programme was created with the objective of enhancing functioning in patients who are bipolar impaired. During the sessions, patients are trained in the use of neurocognitive skills (attention, memory and executive functions) to be applied during their daily routines to improve functional outcome. ${ }^{7,8}$ A recent multicentre, randomised controlled trial (RCT) confirmed the short-term efficacy of functional remediation. ${ }^{9}$ Specifically, it was found that just after finishing the intervention, that is at 6-month follow-up after baseline, the functional remediation group had am improved functional outcome when compared with a treatment-as-usual (TAU) group, especially noted in occupational and interpersonal functioning. ${ }^{9}$ The present study is a continuation of the abovementioned trial. ${ }^{9}$ In this regard, we analysed the functional outcome of the participants 6 months after finishing the therapy, that is 1 year after baseline, in order to study whether functional improvement is maintained over time. As a secondary objective, we aimed to analyse changes in neuropsychological performance.

\section{Method}

This is the 1-year follow-up study of a published RCT that analysed the efficacy of functional remediation at 6-month follow-up. ${ }^{9}$ The project is a multicentre, randomised, rater-masked out-patient trial conducted between 2009 and 2011. It included three parallel arms (1:1:1) to evaluate the efficacy of functional remediation as an add-on therapy compared with psychoeducation and TAU in bipolar disorder. Randomisation was accomplished with the use of a computer-generated sequence. The trial was registered with Clinicaltrials.gov: NCT01370668 and the current analyses were pre-planned in the original protocol.

\section{Participants}

As previously described in Torrent and colleagues, ${ }^{9} 239$ participants with euthymic bipolar I and II disorder were recruited in different centres across Spain. Participants were between 18 and 55 years old and presented a moderate to severe degree of functional impairment (Functioning Assessment Short Test $(\text { FAST })^{10}$ total score $\geqslant 18$ ). All participants were euthymic for at least 3 months before study enrolment, assessed using the Hamilton Rating Scale for Depression (HRSD $\leqslant 8$ ), ${ }^{11,12}$ and Young Mania Rating Scale (YMRS $\leqslant 6$ ). ${ }^{13,14}$ Participants were excluded if they presented any of the following conditions: (a) IQ <85; (b) any neurological or comorbid psychiatric condition affecting neuropsychological performance (patients with neurological diseases, such as epilepsy, or any Axis I comorbidity); (c) electroconvulsive therapy within the past year. All patients provided written informed consent. Ethical approval for the study was granted by the ethics committee at every hospital involved in the study.

\section{Clinical and psychosocial assessments}

At baseline and at 1-year follow-up all participants were evaluated with the following rating scales: YMRS to assess manic symptoms; ${ }^{13,14}$ HRSD to assess depressive symptoms; ${ }^{11,12}$ and 
the FAST scale ${ }^{10}$ to assess psychosocial functioning (a 24-item scale assessing disability in patients with bipolar disorder). FAST assesses six functional domains: autonomy (the capacity to make decisions and do things by oneself), occupational functioning (the capacity to maintain a paid job, efficiency of performing tasks at work, working in the field in which the patient was educated and earning according to the level of the employment position), cognitive functioning (the ability to concentrate, perform simple mental calculations, solve problems, and learn and recall new information), financial issues (the capacity to manage one's finances), interpersonal relationships (relations with friends and family, involvement in social activities, sexual relationships and the ability to defend one's own interests) and leisure time (the capacity to engage in sports or physical activities and to enjoy hobbies). Higher scores indicate a higher degree of functional impairment. The FAST total score was the primary outcome measure defined as the mean change on the total score from baseline to end-point (week 52).

Finally, clinical and demographic variables were collected at baseline and at the end-point through clinical interview and revision of clinical records. They included age, gender, education level, occupational status, body mass index, diagnosis, number and type of episodes, illness duration, age at first hospital admission, age at illness onset, number of admissions to hospital, number of suicide attempts, history of psychosis, pharmacological treatment and adherence, family psychiatric history, comorbidities and several course specifiers, such as rapid cycling, atypical symptoms, melancholia and psychotic depression.

\section{Neuropsychological assessment}

All individuals completed at baseline (week 0) and at follow-up (week 52) a comprehensive neuropsychological battery grouped into six cognitive domains: processing speed, working memory, executive functions, verbal learning/memory, visual memory and attention. For a more detailed description of the neuropsychological battery see Torrent et al. ${ }^{9}$

\section{Interventions}

\section{Functional remediation}

The functional remediation programme consisted of 21 weekly sessions, each lasting $90 \mathrm{~min}$. This intervention addresses neurocognitive issues such as attention, memory and executive functions, but it focuses even more on enhancing functioning in daily routine. The content of the intervention is based on ecological tasks to be performed in two settings, in the clinic as well as at home. Participants receive training with exercises for memory, attention, problem-solving and reasoning, multitasking and organisation to improve their functional outcome. Most of the techniques were based on paper-and-pencil tasks and group activities. (For detailed information on the rationale of this intervention see Martinez-Aran et $a l^{7}$ and Bonnin et al. ${ }^{8}$ ) A manual of the intervention has been published. ${ }^{15}$

\section{Psychoeducation}

The psychoeducation also consisted of 21 weekly sessions of $90 \mathrm{~min}$ each, aimed at preventing recurrences of bipolar illness by improving four main issues: illness awareness, treatment adherence, early detection of prodromal symptoms of relapse and lifestyle regularity. ${ }^{16}$

\section{Treatment as usual}

In the TAU group, participants received prescribed pharmacological treatment without any adjunctive psychosocial therapy.

\section{Statistical analyses}

Sociodemographic and clinical features, at baseline and at follow-up, were analysed using ANOVA for continuous variables and chisquare for categorical variables. Group $\times$ time effects for overall psychosocial functioning (FAST total score), which was the primary outcome of the study, was analysed using repeated-measures ANOVA. This analysis only takes into account complete cases; hence missing values because of loss to follow-up were not analysed.

Secondary outcomes, such as neuropsychological changes were also analysed: verbal learning/memory, executive functions, processing speed, working memory, attention and visual memory. Working memory and processing speed changes were studied using the IQ index provided in the manual by Wechsler (Wechsler Adult Intelligence Scale-III). ${ }^{17}$ To study verbal memory, executive functions and attention, three composite scores were created. These variables were calculated by converting the participants' raw scores to standardised $z$-scores. In turn, these $z$-scores were summed and then averaged in each domain to provide a single score. The verbal memory composite score was composed of the variables of two tests: the California Verbal Learning Test ${ }^{18}$ and the Logical Memory Scale (WMS-III) for learning/memory. ${ }^{19}$ The executive composite score was calculated based on four representative subtests measuring executive function: Stroop Interference, Wisconsin Card Sorting Test (WCST) ${ }^{20}$ perseverative errors, WCST categories and Trail Making Test-part B. ${ }^{21}$ Attention composite score was calculated based on several measures of the Continuous Performance Test (CPT-II) ${ }^{22}$ such as: omission and commission errors, d' attentiveness, beta and reaction time. Finally, exploratory Pearson correlations in each group were conducted to identify clinical and neuropsychological variables associated with functional change during 1-year follow-up. All analyses were two-tailed with alpha set at $P<0.05$ and were performed with the Statistical Package for Social Sciences version 18.

\section{Results}

\section{Patient flow}

Figure 1 represents a chart of the patient flow through the study, from the screening phase to the 12-month follow-up. Of 239 participants at baseline, 172 completed the follow-up phase (72\%); hence, during the intervention (from week 0 to 21) 54 people discontinued treatment and at the end-point (at week 52) 13 further people withdrew from the study. Reasons were, particularly, lost to follow-up or refusing re-evaluation; however, analysis revealed that completers at the end of follow-up did not differ between groups $\left(\chi^{2}=0.199, P=0.9\right)$ : functional remediation $(n=54)$, psychoeducation $(n=60)$ and TAU $(n=58)$. No significant differences between the three arms (functional remediation, psychoeducation and TAU) with regard to number of relapses were found at follow-up $(F=1.05, P=0.349)$ : for the functional remediation (mean 0.67 , s.d. $=2.25$ ), psychoeducation (mean 0.92, s.d. $=2.58$ ) and TAU groups (mean 0.40, s.d. =0.98). Finally, an additional analysis comparing missing cases with complete cases revealed that both groups did not differ in baseline characteristics; hence missing cases were considered 'missing at random'. 


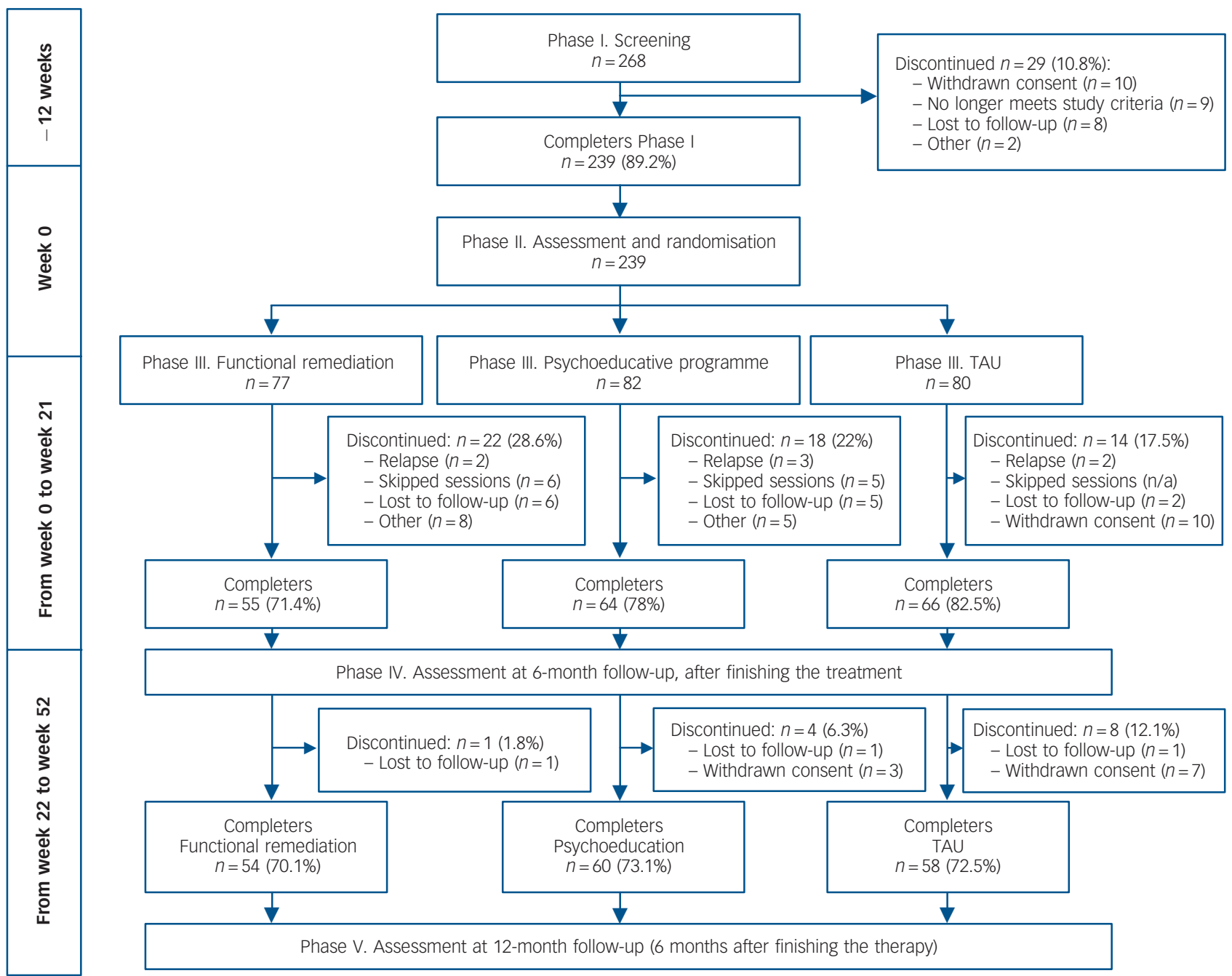

Fig. 1 Flow chart of patients during the study.

$\mathrm{TAU}$, treatment as usual.

\section{Demographic and clinical features}

As shown in Table 1, the three patient groups did not differ on demographic or clinical data at baseline, except for chronicity (illness duration) $\left(F_{(2,230)}=3.2, P=0.04\right)$. Tukey post hoc analyses revealed a significant difference when psychoeducation and TAU were compared $(P=0.02)$. In this regard, the TAU group presented with longer duration of illness when compared with the psychoeducation group. On average, at baseline, participants in the three groups were 40 years old $\left(F_{(2,233)}=0.8, P=0.4\right)$. They were equivalent in terms of educational level $\left(F_{(2,231)}=0.2, P=0.5\right)$ but estimated premorbid IQ showed a trend to be different in the three groups $\left(F_{(2,231)}=2.4, P=0.08\right)$. However, Tukey post hoc analyses revealed that there were no differences between the three groups, only a trend was detected when TAU and psychoeducation were compared $(P=0.07)$ with higher IQ in the TAU group.

\section{Group differences in longitudinal change}

Primary outcome: change in psychosocial functioning

Longitudinal repeated-measures analyses addressing the treatment effect on the primary outcome measure showed significant differences between the three groups (Pillai's trace 0.032, $F=3.071$, d.f. $=2, P=0.049$ ), suggesting an interaction between treatment allocation and time (from baseline to follow-up) and a positive effect of functional remediation over time if compared with psychoeducation and TAU. In Fig. 2, the FAST total score in the functional remediation group was significantly reduced from 30.94 (s.d. $=10.24)$ at baseline to $24.71($ s.d. $=13.60)(t=4.26$, $P<0.01)$ at 12 -month follow-up indicating a significant improvement in global functioning. Between-group effect sizes were small when functional remediation was compared with TAU (Cohen's $d=0.18$ ). The within-group effect size in the functional remediation group was moderate (Cohen's $d=0.49$ ).

Since baseline differences were detected in illness duration (chronicity) and a trend was detected in premorbid IQ, we decided to control for these two potential confounding variables. Repeated-measures ANCOVA comparing the three groups revealed that the model was still significant, showing that patients in the functional remediation group had a better outcome than patients in the psychoeducation or TAU group (Pillai's trace $0.036, F=3.38$, d.f. $=2, P=0.036$ ).

When analysing each of the FAST domains in detail, only one of them, autonomy, was found to be significantly different between groups in favour of the functional remediation group $(F=5.54$, d.f. $=2, P=0.005$, Fig. 3).

Even though subsyndromal depressive symptoms did not differ significantly between the three groups at follow-up, it was 


\begin{tabular}{|c|c|c|c|c|c|c|}
\hline Demographic and clinical variables & $\begin{array}{l}\text { Functional remediation } \\
\text { group }(n=77)\end{array}$ & $\begin{array}{l}\text { Psychoeducation } \\
\text { group }(n=82)\end{array}$ & $\begin{array}{l}\text { TAU group } \\
\quad(n=80)\end{array}$ & $F$ & $\chi^{2}$ & $P$ \\
\hline Age, years: mean (s.d.) & $40.59(9.10)$ & $39.25(8.85)$ & $40.47(8.69)$ & 0.8 & & 0.4 \\
\hline Educational level, years: mean (s.d.) & $12.64(4.06)$ & $13.27(3.66)$ & $13.22(3.54)$ & 0.2 & & 0.5 \\
\hline Estimated premorbid IQ, mean (s.d.) & $105.92(12.51)$ & $103.20(11.63)$ & $107.66(14.31)$ & 2.4 & & 0.08 \\
\hline Age at onset, years: mean (s.d.) & $25.76(8.46)$ & $26.83(8.61)$ & $24.29(7.69)$ & 1.7 & & 0.1 \\
\hline Chronicity, illness duration: mean (s.d.) & $14.83(9.69)$ & $12.69(8.63)$ & $16.38(8.79)$ & 3.2 & & 0.04 \\
\hline Total episodes, mean (s.d.) & $11.86(12.54)$ & $9.93(12.13)$ & $13.03(12.27)$ & 1.3 & & 0.2 \\
\hline Hospital admissions, mean (s.d.) & $2.79(3.64)$ & $2.56(2.51)$ & $2.51(2.26)$ & 0.1 & & 0.8 \\
\hline Hamilton Rating Scale for Depression score, mean (s.d.) & $4.09(2.51)$ & $4.14(2.68)$ & $4.32(2.59)$ & 0.1 & & 0.8 \\
\hline Young Mania Rating Scale score, mean (s.d.) & $1.43(1.83)$ & $1.68(2.12)$ & $1.32(1.77)$ & 0.9 & & 0.4 \\
\hline Women, $n(\%)$ & $44(57.1)$ & $48(58.5)$ & $46(57.5)$ & & 0.03 & 0.9 \\
\hline Lifetime psychotic symptoms, yes: $n$ (\%) & $47(61)$ & $51(62.2)$ & $55(68.8)$ & & 1.2 & 0.5 \\
\hline Lifetime rapid cycling, yes: $n$ (\%) & $12(15.6)$ & $10(12.2)$ & $15(18.8)$ & & 1.6 & 0.4 \\
\hline Family history of affective disorders, $n$ (\%) & $45(58.4)$ & $50(61)$ & $48(60)$ & & 0.01 & 0.9 \\
\hline
\end{tabular}

found that at the end of follow-up, 35 people had subsyndromal symptoms at the time of the assessment presenting scores of between 9 and 16 on the HRSD and between 7 and 11 on the YMRS. Moreover, seven people at the end of follow-up fulfilled criteria for an acute episode, and one of these refused to continue the study. As a consequence of this, we decided to apply repeatedmeasures ANCOVA controlling for the potential confounding effect of subsyndromal symptomatology on functioning. It was found that the improvement in autonomy was still significant; however, changes in the FAST total score were no longer significant after controlling for subthreshold depressive symptoms.

\section{Secondary outcome: neuropsychological changes}

Concerning neuropsychological performance, repeated-measures analyses showed an improvement in verbal memory (verbal memory composite score) in participants who were enrolled in the functional remediation group compared with the other two treatment groups $(F=5.31$, d.f. $=2, P=0.006)$. Once again, we repeated the same analyses controlling for those potential confounding variables identified at baseline (chronicity and premorbid IQ), and we found that the difference in the verbal memory composite score was still significant, favouring the

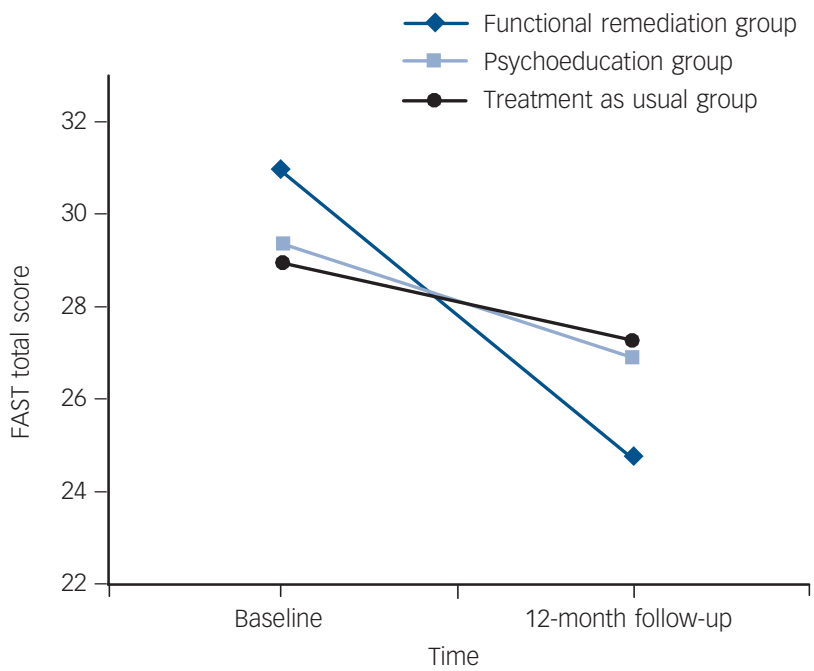

Fig. 2 Functional improvement at 12-month follow-up: Functioning Assessment Short Test (FAST) total score. functional remediation group when compared with psychoeducation and TAU (Pillai's trace $0.087, F=7.64$, d.f. $=2, P=0.001$ ).

Finally, no differences were found in the remaining neuropsychological variables such as composite executive score $(F=0.03$, d.f. $=2, P=0.96)$; processing speed $(F=0.4$, d.f. $=2, P=0.66)$; working memory $(F=1.18$, d.f. $=2, P=0.30)$ or attention composite score $(F=0.6$, d.f. $=2, P=0.52)$.

\section{Relationship between clinical variables and outcome variables}

To investigate the candidate variables associated with functional changes across time, exploratory correlations were run in each of the patient groups. Specifically, correlations were conducted between changes in the FAST total score, changes in neurocognitive domains (verbal memory composite score, executive composite score, working memory index, and processing speed index and attention) and clinical changes (current symptomatology, number of relapses) during 1-year follow-up. Since not all participants were euthymic at the end of the study and in order to avoid confounding effects of symptomatology, the correlations were run only with asymptomatic patients at the end of the study. Hence, the distribution of euthymic participants, defined as HRSD $\leqslant 8$ and YMRS $\leqslant 6$, in each group was as follows:

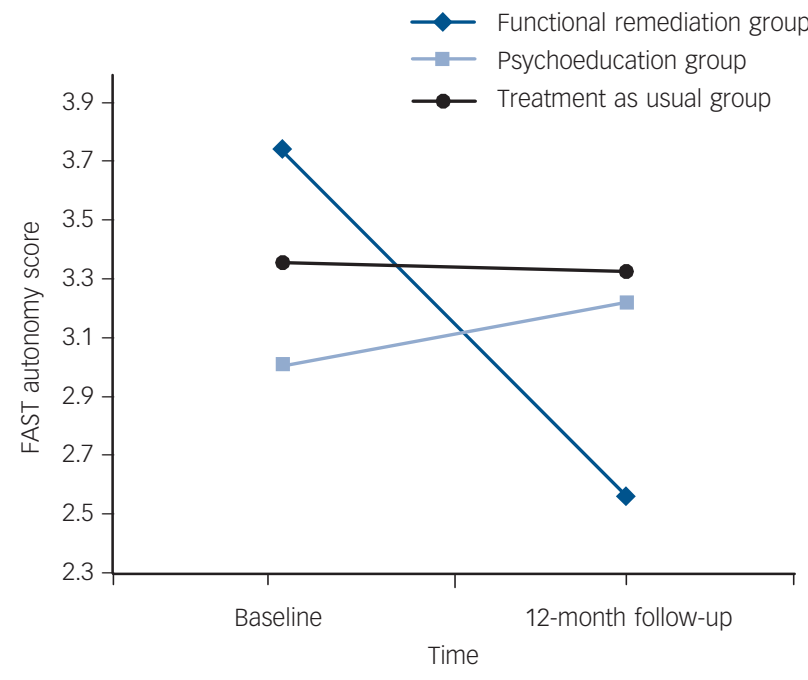

Fig. 3 Improvement of the score in the Functioning Assessment Short Test (FAST) autonomy domain at 12-month follow-up. 


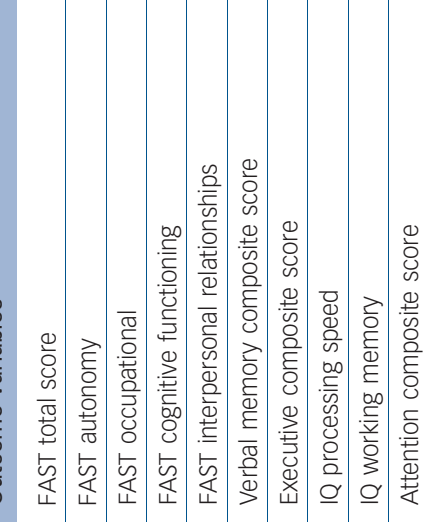

functional remediation $n=49$, psychoeducation $n=48$ and TAU $n=52$.

A significant correlation was found between change in the FAST total score (FAST baseline-FAST end-point) and change in verbal memory composite score (verbal composite baselineverbal composite end-point) in the functional remediation group $(r=-0.32, P=0.039)$. With regard to the psychoeducation group, a positive correlation $(r=0.47, P=0.001)$ was found between changes in the FAST total score and changes in HRSD scores (from 0 to 8 ). Finally, in the TAU group no significant correlations were found between changes in psychosocial functioning and the above-mentioned variables.

\section{Discussion}

\section{Main findings}

The main finding of this study is the confirmation that functional remediation remains effective over time, particularly after 6 months of no further therapy. The participants enrolled in the functional remediation group showed better psychosocial performance than participants in the psychoeducation and TAU groups after 1-year follow-up from baseline. The FAST total score was reduced at the end-point ( 52 weeks) from baseline indicating a clinically and statistically significant improvement in general functioning in the functional remediation group.

As a second finding, a significant enhancement in autonomy was observed at 1-year follow-up, but only in those receiving functional remediation. Autonomy refers to the capacity of patients to do things alone and making their own decisions with more self-responsibility. This includes a basic management of the household, the ability to live alone, to do the shopping and to organise a list of products without requiring additional help. Of note, the psychoeducation group did not improve on functional outcomes significantly, but the main aim of psychoeducation is to prevent relapses, not to improve functioning. The psychoeducation group was used as an intensity-matched comparator to the functional remediation. Although psychoeducation may improve functioning over the long term, because it is effective in preventing recurrences according to Colom and colleagues, ${ }^{16}$ a 1-year follow-up may be too short a time to detect improvement in functional outcome in those who received psychoeducation. Moreover, the present study selected patients who were highly impaired at baseline with a FAST total score $\geqslant 18$, and psychoeducation may be much more effective in patients who are not functionally impaired.

\section{Improvements in autonomy}

With the functional remediation intervention we aimed to reduce the difficulties that people with bipolar disorder present in their daily life because of their illness, providing them with some tools to improve their ability to solve daily problems. In the primary 6-month end-point analysis patients improved in their adaptation and occupational performance as well as in their interpersonal relationships. ${ }^{9}$ Six months after finishing the intervention, the improvement in these two areas (occupational and interpersonal relationships) disappeared and only autonomy was found to be significant at 12-month follow-up. There are a number of reasons this may be the case: the most likely one is that because of loss to follow-up the study is statistically underpowered, at least, to detect differences when analysing the different FAST domains. Another possible reason is that functional remediation needs booster sessions to maintain the effects of the intervention in the long term, since it seems that results were stronger at 6-month follow-up than at 12-month follow-up. 


\section{Improvements in verbal memory}

Regarding neurocognitive performance after 1 year, significant improvement in verbal memory was detected. Our data suggest that there is a positive relationship between improved verbal memory and psychosocial functioning that was confirmed with the significant correlation found between changes in the FAST total score and verbal memory in the functional remediation group. This is important since verbal memory and executive functions have been identified as predictors of global functioning. ${ }^{2,3,23}$ Therefore, by improving these cognitive processes patients are better equipped with the necessary underlying skills to find greater success in their daily activities that most often require adequate cognitive functioning. Functional remediation can target, as a secondary objective, the cognitive deficits of patients with bipolar disorder, yielding not only better neuropsychological skills but also a generalised improvement in functional abilities, such as the before-mentioned autonomy. However, results have to be interpreted with caution as a learning effect in the tests related to verbal memory cannot be excluded either, because of a lack of parallel tests. However, verbal memory improvements were observed only in the functional remediation group.

\section{Findings from other studies}

Despite the well-characterised association between neurocognitive deficits and functional outcome in patients with euthymic bipolar disorder, only three trials so far have addressed the efficacy of neurocognitive-based psychosocial interventions. The first one is a small, uncontrolled trial of 18 participants with bipolar disorder showing an improvement in psychosocial and occupational functioning. ${ }^{24}$ The second study is a multicentre, randomised, rater-masked clinical trial showing the efficacy of functional remediation in improving psychosocial functioning, which is the first part of the present study. ${ }^{9}$ Recently, another randomised trial has been registered aiming to evaluate the effects of cognitive remediation in 40 participants with bipolar disorder in full or partial remission with persistent cognitive difficulties, but results are still not available. ${ }^{25}$ Similarly, Bauer and colleagues ${ }^{26}$ found that collaborative care, a psychosocial intervention, showed a positive effect on functioning in the long term. However, this intervention was not based on a neurocognitive intervention; instead it was based on three different components that addressed the patient, provider and systems aspects of care. This suggests that there might be different paths to achieve functional improvement in patients with bipolar disorder, ranging from neurocognitive-based interventions to community-care programmes. Altogether, it highlights the need to study the long-term efficacy of the programmes aiming to improve functional outcome in those with bipolar disorder. $^{27}$

\section{Future directions}

The above-mentioned studies, and particularly the functional remediation studies, have just started to be explored in bipolar disorder, so that the cognitive domains to be trained; the feasibility of the programme, including number and frequency of session; duration and intensity of the intervention warrant further investigation. Moreover, other relevant variables may influence results, such as age of patients, chronicity or severity of illness (such as number of episodes). Patients included in this study were chronic, with an average age of 40 years. Probably younger patients, earlier in the course of the illness, may have greater potential for cognitive and functional improvement. For these reasons, the development of interventions aiming at preventing neurocognitive and functional impairment in the early stages of the illness are urgently needed.
Moreover, further studies should address the identification of the moderators and mediators in the prediction of functional outcome because this may help to disentangle the complex network of variables that contribute to functional outcome in bipolar disorder. ${ }^{28}$ In fact, a recent study found that patients' composite verbal memory score explained partially the variability of functional outcome (measured by means of FAST) at 6-month follow-up. Verbal memory had a significant indirect effect on functional outcome, partly mediating the relationship between depressive symptoms and functional status. ${ }^{29}$ Another line of future research is that further studies will have to confirm the duration of the effects of the intervention and to evaluate whether or not the inclusion of some regular booster sessions may help to maintain the effects of the intervention longer term. Finally, computerised neurocognitive training is also needed. So far, little evidence exists of neuropsychological computerised programs for patients with bipolar disorder. A recent study with a sample of affective patients including some with bipolar disorder has been published with positive results (improvement neuropsychological and clinical outcome).$^{30}$ In this regard, functional remediation may be a promising tool in combination with other psychosocial interventions to improve functional outcome.

\section{Strengths and limitations}

The most relevant limitations of the study are that the follow-up is still relatively short and the study lost power because of the small sample size; in light of this, missing cases were excluded for the analyses and considered 'missing at random'. However, the study provides strong evidence that improvements in psychosocial functioning can be achieved and maintained beyond the end of a structured psychological intervention with emphasis on neurocognitive strategies. The randomised, rater-masked design and the presence of not only a TAU but also a psychoeducation arm provides further strength to the findings. Establishing how to adjust and implement interventions such as functional remediation for people with bipolar disorder in the community and how to make them cost-effective is the next step forward.

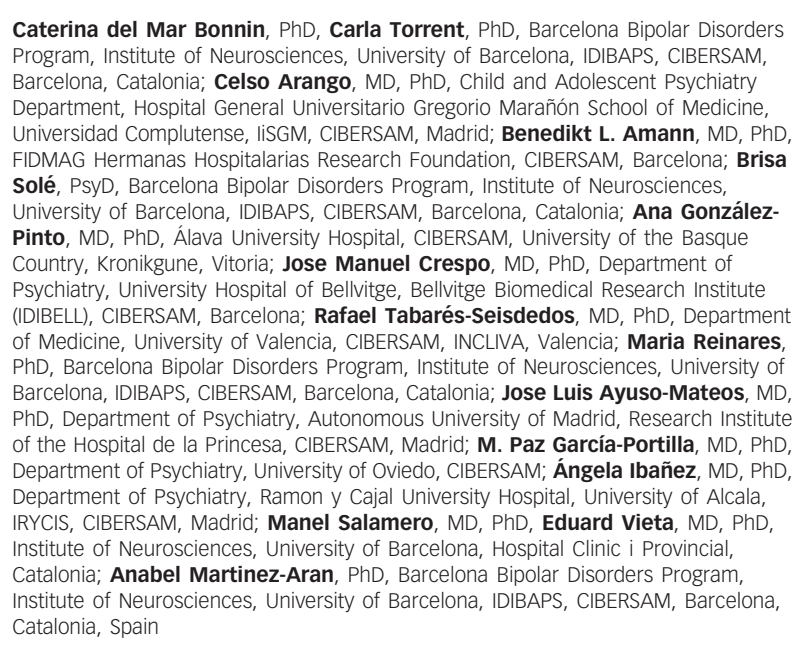
Catalonia, Spain

Correspondence: Eduard Vieta, Bipolar Disorder Program, Clinical Institute of Neuroscience, Hospital Clinic of Barcelona, Villarroel, 170. 08036 Barcelona, Spain. Email address: evieta@clinic.ub.es

First received 24 Jul 2014, final revision 15 Dec 2014, accepted 25 Jan 2015

\section{Funding}

This study was supported by an ETES grant from the Spanish Ministry of Economy and Competitiveness (PI080180 \& PI08/90825, PI08/90327, PI08/90675, PI08/90224, PI08/90654, PI08/90189, PI08/90916, PI08/90416, PI08/90094). PI11/00637, PI12/00912, integrado en el 
Plan Nacional de I+D+I y cofinanciado por el ISCIII-Subdirección General de Evaluación y el Fondo Europeo de Desarrollo Regional (FEDER)", the CIBERSAM and the Comissionat per a Universitats i Recerca del DIUE de la Generalitat de Catalunya to the Bipolar Disorders Group (2014 SGR 398).

M.R. was supported by Beatriu de Pinós, Secretaria d'Universitats i Recerca de Departament d'Economia i Coneixement, de la Generalitat de Catalunya i del programa COFUND de les Accions Marie Curie del 7è Programa marc de recerca i desenvolupament tecnològic de la Unió Europea. M.R.-C. (CIBERSAM Functional Remediation Group) was supported by a Sara Borrell Health Research Fellowship from the Institute of Health Carlos III, Spanish Ministry of Economy and Competitiveness, an Alicia Koplowitz Research Grant and Alicia Koplowitz Grant for Short-Term Placements from the Alicia Koplowitz Foundation (Madrid, Spain).

\section{Acknowledgements}

The CIBERSAM Functional Remediation Group consists of: Analucía Alegría, Susana Al-Halabi Silvia Alonso-Lana, Celia Anaya, Jesus Valle, Vicent Balanzá-Martínez, Francesc Colom, Sara Barbeito, Julio Bobes, Ana Calvo, Esther Cerrillo, Patricia Correa, Núria Custal, Patricia Fernández Miryam Fernández, Inmaculada Fuentes-Durá, Gonzalo Galván, Sandra Isella Ramon Landín-Romero, María Mayoral, José Manuel Menchón, Jessica Merchan-Naranjo, Esther Jiménez, Jordi Ortiz-Gil, Isabella Pacchiarotti, Adriane R Rosa, Marta Rapado-Castro, Esther Jiménez, Jordi Ortiz-Gil, Isabella Pacchiarotti, Adriane R Rosa, Marta Rapado-Castro,
José-María Rodao, Pilar A Saiz, Jose Sánchez-Moreno, Bárbara Segura, Gabriel Selva, Jerónimo Saiz-Ruiz, Marta Subirá, Imma Torres, Amaia Ugarte, Patricia Vega.

\section{References}

1 Rosa AR, Reinares M, Michalak EE, Bonnin CM, Sole B, Franco C, et al. Functional impairment and disability across mood states in bipolar disorder. Value Health 2010; 13: 984-8.

2 Martino DJ, Marengo E, Igoa A, Scápola M, Ais ED, Perinot L, et al. Neurocognitive and symptomatic predictors of functional outcome in bipola disorders: a prospective 1 year follow-up study. J Affect Disord 2009; 116: 37-42.

3 Bonnin CM, Martinez-Aran A, Torrent C, Pacchiarotti I, Rosa AR, France C, et al. Clinical and neurocognitive predictors of functional outcome in bipolar euthymic patients: a long-term, follow-up study. J Affect Disord 2010; 121 156-60

4 Martinez-Aran A, Vieta E, Torrent C, Sanchez-Moreno J, Goikolea JM Salamero $\mathrm{M}$, et al. Functional outcome in bipolar disorder: the role of clinica and cognitive factors. Bipolar Disord 2007; 9: 103-13.

5 Tabares-Seisdedos R, Balanza-Martinez V, Sanchez-Moreno J, Martinez-Aran A Salazar-Fraile J, Selva-Vera G, et al. Neurocognitive and clinical predictors of functional outcome in patients with schizophrenia and bipolar I disorder at one-year follow-up. J Affect Disord 2008; 109: 286-99.

6 Lewandowski KE, Cohen BM, Keshavan MS, Sperry SH, Ongür D. Neuropsychological functioning predicts community outcomes in affective and non-affective psychoses: a 6-month follow-up. Schizophr Res 2013; 148 34-7.

7 Martinez-Aran A, Torrent C, Sole B, Bonnin M, Rosa AR, Sanchez-Moreno J, et al. Functional remediation for bipolar disorder. Clin Pract Epidemiol Ment Health 2011; 7: 112-6.

8 Bonnin CM, Torrent C, Vieta E, Martínez-Arán A. Restoring functioning in bipolar disorder: functional remediation. Harv Rev Psychiatry 2014; 22: 326-30

9 Torrent C, Bonnin CM, Martinez-Aran A, Valle J, Amann BL, González-Pinto A et al. Efficacy of functional remediation in bipolar disorder: a multicenter randomized controlled study. Am J Psychiatry 2013; 170: 852-9.

10 Rosa AR, Sanchez-Moreno J, Martinez-Aran A, Salamero M, Torrent C, Reinares M, et al. Validity and reliability of the Functioning Assessment Short Test (FAST) in bipolar disorder. Clin Pract Epidemol Ment Health 2007; 3: 5 .
11 Hamilton M. A rating scale for depression. J Neurol Neurosurg Psychiatry 1960; 23: 56-62.

12 Ramos-Brieva JA, Cordero-Villafáfila A. Validation of the Castillian version of the Hamilton Rating Scale for Depression [in Spanish]. Actas Luso Esp Neurol Psiquiatr Cienc Afines 1986; 14: 324-34.

13 Young RC, Biggs JT, Ziegler VE, Meyer DA. A rating scale for mania: reliability, validity and sensitivity. Br J PSychiatry 1978; 133: 429-35.

14 Colom F, Vieta E, Martinez-Aran A, Garcia-Garcia M, Reinares M, Torrent C, et al. Spanish version of a scale for the assessment of mania: validity and reliability of the Young Mania Rating Scale [in Spanish]. Med Clin (Barc) 2002; 119: $366-71$.

15 Vieta E, Torrent C, Martinez-Aran A. Functional Remediation for Bipolar Disorder. Cambridge University Press, 2014.

16 Colom F, Vieta E, Martinez-Aran A, Reinares M, Goikolea JM, Benabarre A et al. A randomized trial on the efficacy of group psychoeducation in the prophylaxis of recurrences in bipolar patients whose disease is in remission. Arch Gen Psychiatry 2003; 60: 402-7.

17 Wechsler D. The Wechsler Adult Intelligence Scale - III (WAIS-III). Harcourt Assessment, 1997.

18 Delis DC, Kramer JH, Kaplan E, Ober BA. California Verbal Learning Test. Psychological Corporation, 1987.

19 Wechsler D. Wechsler Memory Scale (3rd edition). Harcourt Assessment 1997.

20 Heaton RK. Wisconsin Card Sorting Test Manual. Psychological Assessment Resources, 1981.

21 Reitan RM. Validity of the Trailmaking Test as a indication of organic brain damage. Percept Mot Skills 1958; 8: 271-6.

22 Conners CK. Conners' Continous Performance Test (CPT-II). Technical Guide and Software Manual (2002 edn). Multihealth Systems, 2002.

23 Laes JR, Sponheim SR. Does cognition predict community function only in schizophrenia? A study of schizophrenia patients, bipolar affective disorder patients, and community control subjects. Schizophr Res 2006; 84: 121-31.

24 Deckersbach T, Nierenberg AA, Kessler R, Lund HG, Ametrano RM, Sachs G, et al. RESEARCH: Cognitive rehabilitation for bipolar disorder: an open trial for employed patients with residual depressive symptoms. CNS NeurosCi Ther 2010; 16: 298-307.

25 Demant KM, Almer GM, Vinberg M, Kessing LV, Miskowiak KW. Effects of cognitive remediation on cognitive dysfunction in partially or fully remitted patients with bipolar disorder: study protocol for a randomized controlled trial. Trials 2013; 14: 378.

26 Bauer MS, McBride L, Williford WO, Glick H, Kinosian B, Altshuler L, et al. Collaborative care for bipolar disorder: part II. Impact on clinical outcome, function, and costs. Psychiatr Serv 2006; 57: 937-45

27 Fuentes-Dura I, Balanza-Martinez V, Ruiz-Ruiz JC, Martínez-Arán A, Girón M, Sole $B$, et al. Neurocognitive training in patients with bipolar disorders: current status and perspectives. Psychother Psychosom 2012; 81: 250-2.

28 Vieta E. The bipolar maze: a roadmap through translational psychopathology. Acta Psychiatr Scand 2014; 129: 323-7.

29 Bonnin CM, Gonzalez-Pinto A, Sole B, Reinares M, González-Ortega I, Alberich $S$, et al. Verbal memory as a mediator in the relationship between subthreshold depressive symptoms and functional outcome in bipolar disorder. J Affect Disord 2014; 160: 50-4.

30 Preiss M, Shatil E, Cermakova R, Cimermanová D, Ram I. Personalized cognitive training in unipolar and bipolar disorder: a study of cognitive functioning. Front Hum Neurosci 2013; 7: 108. 